\title{
What Counts as a 'Good' Metaphysical Language?
}

\section{JTM Miller}

\section{Pre-proof version - please cite final published version}

One recent metaontological debate focuses on the significance of alternative languages. We talk about tables, as composite objects, but it is possible for there to be a linguistic community that substitutes our table-talk for talk of particles-arranged-tablewise. The deflationist thinks that this shows that some ontological debates are non-substantive. The possible language is no better than our actual language for the purposes of metaphysics, so if it is true for us that there are tables, this is due to nothing more than the shallow fact that we happen to speak English. The realist, by contrast, thinks that among the possible languages some are metaphysically better than others. ${ }^{1}$ For the realist, we can settle the metaphysical debate about the existence of tables by identifying which is the best of the candidate languages, and determining whether tables exist according to that language.

Claims that there are multiple 'equally good' languages to describe the world and that we cannot compare those languages to see which is the best can be found in throughout the metaontological deflationist literature. ${ }^{2}$ But, both the deflationist and the realist rely on qualitative comparisons of languages: the deflationist thinks some are equally good, while the realist thinks that one is best, or that one can be better than another. This leaves us with a question: what determines the metaphysical 'goodness' of a language?

This kind of normative question is not new to philosophy. Attempts to define the 'good' have been a central part of meta-ethics since Moore's argument that the most important question in ethics is 'how is 'good' to be defined?' (Moore [1903] 1993: 58). The focus of this paper is to consider, in a sense, that same question but in the context of the debate between metaontological realists and deflationists. My contention in this paper is that the debate turns significantly on what each side mean by 'good' or 'better' when they say that one metaphysical language can be 'better' or that all languages are 'equally good'. More specifically, I will argue that there is a way in which at least some deflationists and realists are talking past each other, employing different normative notions to compare languages that, I argue, the attempt to deflate metaphysical arguments in this way fails to hit its intended mark.

\footnotetext{
1 I use the terms 'objectively best language', 'metaphysically privileged language' (or just 'privileged'), and 'fundamental language' interchangeably here.

2 Sider (2009: 3 fn.10) identifies, as well as what he calls a 'silent majority watching from the sidelines' the following: Carnap (1950); Chalmers (2009); Hirsch (2002a, 2002b, 2005); Peacocke (1988); Putnam (1975, 1987); Sidelle (2002); Sosa (1999); Thomasson (2007, 2008, 2009, 2015).
} 
To make this argument, I will make use of the concept of 'semantic purpose' that is present in the metaontological realist work of Sider (2011), and used in an argument in favour of deflationism by Irmak (2013). 'Semantic purpose' will be used as a way to understand what languages are for. That is, what they, as languages, are intended to help us capture or describe, and hence provide a criterion against which to judge 'good' those languages are. Semantic purpose is therefore a highly useful concept to understand whether or how languages might be compared to see which is 'best', or to see if languages are really 'equally good'.

After outlining Irmak's argument against Sider, I will argue that the argument rests on too narrow a conception of the semantic purpose of languages proposed by metaphysical theories as being privileged or fundamental. I argue that this undercuts the conclusion that there are 'equally good' languages as this conclusion can only be reached if the semantic purposes of the putative privileged languages are the same.

I argue that if we do wish to consider comparing putative privileged languages, then we should (i) understand the idea of a semantic purpose in a coarse-grained (rather than fine-grained) way; and, (ii) distinguish between two tasks where the first is to decide whether there is an objectively best language, and the second is to debate the content of that fundamental language. I argue that once we have done this, candidate metaphysically privileged languages can, in principle, be compared to see which is metaphysically best. Actually comparing languages will still be very hard. Indeed, if I am right, comparing languages, including for the conclusion that they are equally good, will require will rely on engaging in various debates about what metaphysical principles we accept as we start to create a putative privileged language. However, to debate those principles is to engage in substantive metaphysical disputes, and hence supports a metaontological realist position.

A brief note before the main substance of this paper. A central claim of this paper is that we must be careful to distinguish between arguments that there is a metaphysically privileged language, and arguments about what that language looks like (or how we can find out what it looks like). Beyond agreeing with Sider's metaontological realism, I will be silent about other commitments that he defends, including his use of notions such as 'joint-carving' and his focus on structure. My claims stand independent of whether we wish to accept those commitments.

\section{I}

Irmak (2013) produces a typical reconstruction of an argument for the existence of a privileged or fundamental language, taking his cues from Sider (2011):

' 1 . There are objective similarities and differences 'out there' in the world. 
2. The point of human inquiry is to conform itself to the world in a way that correctly represents these similarities and differences rather than to make or construct them.

3. There might be different languages that state true propositions. But when there are, one must be the better description of reality. A description is better than the others just in case the propositions in that description are cast in joint-carving terms so that its ideology better matches the range of differences in the world.

4. There is an objectively privileged language which best carves nature at the joints and can be used for 'writing the book of the world'.' (Irmak, 2013: 4)

These four theses lead to the argument:

'a) The language that best carves nature at the joints is the best language (From 3 above).

b) The language of physics is the language which best carves nature at the joints.

c) Therefore, the language of physics is the best (most objectively privileged) language.' (Irmak, 2013: 5)

Irmak concludes his reconstruction by stating that:

According to Sider, given that there is a best, objectively privileged language that can describe the world as it is, we can say that ontological questions are substantive because they can be formulated in this fundamental language. An ontological debate when it is done in this fundamental language, then, is a serious, quasi-scientific debate about which answer gives a better description of reality. What is at stake in those debates, in this account, is discovering the most fundamental structure of reality. Even though rival ontologies might state true propositions about the world, one is the best. (2013: 5)

In order to defend the substantivity of metaphysical debates, Sider wishes to defend the possibility and intelligibility of an objectively privileged language. This is a fundamental language, which provides the best description of reality. It is this realism about there being a fundamental language that Irmak focuses their argument on, thereby attempting to undercut the claim of substantivity in metaphysical debates.

\footnotetext{
${ }^{3}$ Note that the language of physics does not only contain 'physical' predicates (leaving aside the question of which predicates should be admitted as being 'physical'). For Sider the best language also contains basic logical notions (such as quantification, negation), plus a predicate for set membership (Sider 2011: 292).
} 
Usefully for the dialectic that I am engaging with here, Irmak also provides an overview of the structure of an argument against Sider (numbers and letters referring to the above quotes):

In what follows I will argue that knee-jerk realism and particularly the claim that the language of physics is objectively privileged are false. I grant (1) and (2) above which assures that there is a mind-independent reality with objective similarities and differences that we try to wrap our minds around. Together they are enough to provide a modest form of realism that many are eager to preserve. I argue that (3) is false and once we reject it we also lose (4). Therefore, I reject (a), which depends on the truth of (3), on the implicit argument for (c); that the language of the physical is objectively privileged. My arguments against (3) and (4) rely on the idea of a semantic purpose. I argue that once we acknowledge the roles of purposes where we introduce new terms to our language (locally) and when we adopt new languages or provide descriptions (holistically), we no longer are able to claim that there is a single fundamental and objectively privileged language. Therefore, I conclude that kneejerk realism is wrong and there is no single best language that one can use to "write the book of the world.' (2013: 5)

Before unpacking this argument in detail, it is worth noting a possible tension in the stated aims. At the beginning of this quote, the aim of the paper is to 'argue that knee-jerk realism and particularly the claim that the language of physics is objectively privileged are false' (my emphasis). Later, it is stated that 'therefore, I conclude that knee-jerk realism is wrong and there is no single best language that one can use to 'write the book of the world' (my emphasis). These two conclusions are clearly not the same. One can hold that there is a single best language that one can use to write the book of the world without thinking that it is the language of physics that is objectively best. I will discuss the importance of how these two claims come apart in relation to broader metaontological issues in later sections.

It is worth stressing here that Irmak's claim is not that there are no objective similarities or differences in reality, or that as humans we do not attempt to express these similarities and differences. For Irmak, we do not create or construct differences and similarities in the world. Or, more precisely, we do not create them all. Some social facts may have objective status, and yet are constructed by individual (or groups) of humans.

Rather, the claim is that there can be no single objectively best language to express these pre-existing objective similarities and differences. This is a crucial commitment, common across the metaontological literature, to what I will call 'minimal realism'. Minimal realism is the view that the world contains some similarities and differences that are entirely independent of us and our language choice, yet we nonetheless are trying to express those differences within our language and general inquiries into the world. This is a conjunction of (1) and (2) above. Crucially, Irmak wishes to hold onto 
these claims, whilst rejecting (3). Irmak is not alone in his acceptance of minimal realism, but rejection of a privileged or objectively best language. Similar views can be found in the highly influential work of Hirsch (2011) and Thomasson (2015). ${ }^{4}$ Whilst Hirsch and Thomasson put forward different versions of neo-Carnapianism, both wish to hold that there are multiple equally good ways to, but no best way to describe the world. ${ }^{5}$

We should note that these aspects of minimal realism might come apart. That it, it may be possible to think that there are objective similarities and differences in the world but that our language is not intended to, or simply cannot, represent them. I leave that form of anti-metaphysical argument aside here, and focus solely on those that would accept minimal realism as the conjunction of these two theses.

As also expressed in the above quote, the argument against theses (3) and (4) comes from the idea of a 'semantic purpose'. Semantic purpose refers to the idea that when we introduce a new term to a language, then we do so for a particular reason. The semantic purpose associated with a new term allows us to fix the meaning of the new term from amongst the infinite number of candidate meanings. We can explain this idea with reference to the word 'inch'. The semantic purpose of introducing this word into our language is to measure the size of smallish things. This purpose could be achieved in a number of different ways, each of which would satisfy this goal equally well. The two sentences,

S: Let 'inch' be that unit such that 15 of them add up to the length of my screen.

S': Let 'inch' be that unit such that 30 of them add up to the length of my computer screen.

pick out instances where we have simply stipulatively defined 'inch' differently. It just so happens that the convention followed in S' defines 'inch' as half the size of the convention followed in defining 'inch' in S. However, what the sentences express is empirically identical. My computer screen is only one length across, and both sentences equally well express this fact. In this case, neither S nor S' carve at the joints better than the other.

We are then told to take the idea of semantic purpose, and generalise it. First, semantic purpose is extended from the conventional based examples such as 'inch' to technical terms within different forms of discourse. Take the term 'elementary particle':

The meaning of this physical term is not fixed conventionally yet the semantic purpose has the same role as in the case of determining the meaning of 'inch': it narrowed down the range of candidate meanings. Had the purpose for introducing

\footnotetext{
4 See Miller (2016) for a response.

5 Irmak's, and other deflationist's, commitment to 'minimal realism' is tied to their claim to be putting forward a realist position, and not a conceptualist, anti-realist, or idealist position. For example Hirsch stresses that his view does not endorse 'an absurd form of linguistic idealism' (2002b; 2011, 70). Here, I accept this claim to be realists; see [author paper] for a response.
} 
the term 'elementary particle' been different and instead of naming the most fundamental physical particle, it was, say, naming the elementary basic unit of life, then DNA, cells, or organs might have been among the candidate meanings for the term 'elementary particle.' The reason why we don't realize that we have semantic purposes when we do science, ethics or economics might be that those purposes are so obvious that they often need no explicit discussion (Irmak, 2013: 7)

In this case, the meaning of the term 'elementary particle' can only be identified given a particular semantic purpose, that of naming the most fundamental physical furniture of the universe. Comparing any candidate meanings requires specifying this purpose. It is only with respect to this purpose that candidate meanings can be seen as better or worse than each other (as is the case for 'elementary particle') or equally good (as is the case for 'inch'). This is an extension from the initial conventionbased notion of semantic purpose, to cover also the introduction of new concepts into more technical languages.

Semantic purpose is subsequently generalised even further, to cover entire languages:

I want to take the idea of semantic purpose seriously and generalize it in the following way: Different choices of candidate meanings for terms (locally) and of different languages and descriptions (holistically) may only be evaluated as better or worse only relative to their purpose (Irmak, 2013: 7, my emphasis)

It is the generalisation of semantic purpose to entire languages that is crucial to these kinds of arguments against the idea of an objectively best language. The argument is that once we recognise that languages, like terms and concepts before, can only be compared relative to the semantic purpose for which they are introduced, then we 'must reject the claim that where we have different languages to describe reality one must be (absolutely) better' (Irmak, 2013: 10).

I confess that I find the notion of a 'semantic purpose' for an entire language unclear and cannot offer any major insights into the idea. It is not obvious at all that the generalisation from how single terms are introduced to how languages are is possible. For instance, one issue is that whilst we can relatively easily isolate a single term within a language and assess its semantic purpose, it is not entirely clear that we can isolate any language from any other language. This is not to support a holism about all languages. It is instead that it is not clear what the semantic purpose of English is opposed to that of French, or that of the language of physics, or the language of economics (etc.). For starters, these languages overlap in semantics, grammar, and what they seek to describe. Irmak's argument relies on there being some way to distinguish between the semantic purposes of languages, but gives us no tools about how to do so. From the discussion, it seems clear that Irmak does not claim that different natural languages might have different purposes. But they do not provide us with a method to know why it is 
that natural languages do not differ in semantic purpose, but the languages of physics and economics do. It is at least possible that natural languages were introduced with different purposes. However, I only note this lack of details, and will leave this to one side in this paper; and assume, with Irmak, that semantic purposes for whole languages can be given.

\section{II}

As we have seen above, the claim is that there is no shared purpose through which we can judge languages so as to see which is the objectively best language. The support for the claim comes in considering an example that Sider (2011) uses in defence of knee-jerk realism - the view that at least some parts of human inquiry aims to conform itself to the world. As Irmak states: '[t]he example is important because it is supposed to show how and why physics is objectively privileged' (2013: 10). Sider's example compares the relative 'betterness' of physics and shmysics, where shmysics is the subject matter of a set of propositions arrived at via an arbitrary function that creates a different interpretation of a set of propositions that express the set of physical truths (of which physics is the subject matter). These two languages share the same purpose: that of explaining the fundamental physical structure of reality, and so Irmak agrees with Sider that in this case physics is the objectively better language.

This kind of assessing of languages is not possible when the languages do not have the same purpose. We are asked to imagine two languages (or sets of propositions) $\mathrm{S}$ and $\mathrm{P}$, where $\mathrm{P}$ is the set of physics propositions, and $\mathrm{S}$ is a set of propositions that express some aesthetic judgements. Say further, for a statement $\mathrm{T}$ there is a corresponding aesthetic proposition in $\mathrm{S}$. But, ' $[\mathrm{c}]$ an we say that $\mathrm{P}$ is better than S? No-it doesn't make sense to say so. Better for what? On what basis will we compare them? We cannot evaluate them by assuming that they both have the same purpose; this is just not the case' (Irmak 2013: 11).

Irmak continues: '[t]hus, there are two possibilities. First, we might say that even though $\mathrm{P}$ and $\mathrm{S}$ are intended to serve different semantic purposes, we can still compare them. Second, and more plausibly, we can concede that P and S are "just different"; they are different ways to describe reality' (2013: 11). Neither option is a good one for anyone that wishes to claim that there is an objectively best language. Therefore, if two sets of propositions $\mathrm{S}$ and $\mathrm{P}$ have different purposes then we cannot objectively compare them.

The question at hand then is: is there a relevant criteria that putative privileged languages share that would allow us to compare them? If there is not, and there are only the different purposes that the different languages have, then Irmak is correct. If there is, then the realist can hold that we can, in principle at least, compare languages to see which is the best. My claim will be that Irmak is wrong to 
say that in relevant cases $\mathrm{S}$ and $\mathrm{P}$ are intended to serve different purposes, and hence the realist can provide a shared purpose to compare them against.

In order to do this, I need to make a distinction between narrow and broad semantic purposes. Narrowly, the semantic purpose of the term 'inch' is to measure small-ish things. However, semantic purposes come with varying degrees of breadth. 'inch' also has the broader semantic purpose to measure all things. Perhaps 'inch' is less good at measuring the distance from here to the moon than the term 'mile' in part because one of the reasons that we introduced the term 'mile' was to measure longer distances and objects.

Let us try to put this more precisely. A semantic purpose is a reason for which a term or language is introduced. Such reasons can vary, and some can be more general, or broad, than others. 'Measuring small-ish things' and 'measuring longer distances' are both semantic purposes that are narrower than 'measuring things', and if we want to compare our use of 'inch' and 'mile' which purpose we compare them against will be relevant to which we think is better. Against the first, 'inch' in better; against the second 'mile' is better; and it is unclear which is better against the third. In this cases, it is plausible to believe that 'inch' and 'mile' are equally good terms relative to the purpose of 'measuring things', unless, perhaps, we have reason to believe that there are more small things than large things, or vice versa.

Let us now apply this to languages. As Irmak defends, we can take the language of physics to have a semantic purpose of representing the objective physical similarities and differences in the world, and a semantic purpose of the language of economics is to represent the objective economic similarities and differences in the world. Are these narrow or broad semantic purposes? This depends on how we look at them. Relative to representing the objective physical similarities and differences in this room, representing the objective physical similarities and differences in the world is a broad semantic purpose. Relative to representing the objective similarities and differences in the world, representing the objective physical similarities and differences in the world is a narrow semantic purpose as it only aims at the physical similarities and differences and we might think that there are non-physical similarities and differences in the world. Similar claims can be made about the language of economics, and its semantic purpose to represent the objective economic similarities and differences in the world, or just those within, say, one country.

Where does this leave us? Irmak's claim is that we cannot compare the language of physics and the language of economics as they have different purposes: representing the objective physical similarities and differences in the world, and representing the objective economic similarities and differences in the world respectively. On this, I agree with Irmak. We cannot compare these languages relative to these semantic purposes and if we do try to we should accept that they are just different. 
What if we just pick one? Relative to the semantic purpose of representing the objective physical similarities and differences in the world, it seems clear, to me at least, that the language of physics is better. Physics represents better physical similarities and differences than economics. In contrast, relative to representing the objective economic similarities and differences in the world, economics is better, as economics represents better economic similarities and differences. ${ }^{6}$ That this is the case should not be surprising. Each of these languages aims to represent those similarities and differences, and those developing the languages have got very good at creating language that fulfil that purpose.

It also gets us nowhere if we want to defend a realism about a metaphysically best language. This is because, we must be careful not to import first-order metaphysical claims into our metametaphysical theorising. We cannot assume what kinds of similarities and differences the world actually contains. We can assume that it contains some, as this was the commitment to minimal realism that was discussed earlier. Minimal realism held that the world contains some similarities and differences that are entirely independent of us and our language choice, yet we nonetheless are trying to express those differences within our language and general inquiries into the world. What we cannot assume that the differences and similarities in the world are physical in nature, nor that they are economic, aesthetic, ethical, modal, or any other kinds of distinction we might think of. ${ }^{7}$ To do so would be to beg the question. If we assume that realist only contains physical similarities and differences, then representing the objective physical similarities and differences and representing all objective similarities and differences collapse into each other. If a language satisfies one, then it must satisfy the other. But we cannot assume that in our metametaphysics.

In light of this, I argue that we can instead take the semantic purpose of a putative privileged language is to represent all objective similarities and differences in the world. The metaphysically best language is introduced in order to represent all the ways that the world really is, however that may be; what such a language is for is to represent all objective similarities and differences in the world, not just some subset of them. Absolutely we cannot compare languages relative to different semantic purposes, but we can compare them relative to the same semantic purpose, and the semantic purpose intended by the metaphysician when introducing terms or a language that they think is best is to represent all similarities and differences in the world.

If we think that the language of physics is the best metaphysical language, then we think that it is a good language both at representing physical similarities and differences in the world and at representing all objective similarities and differences in the world. The same holds, mutatis mutandis for the language

\footnotetext{
6 This is an empirical claim perhaps, but I think a reasonable assumption. Some (reductive) physicalists might deny this assumption, but as I will discuss in detail in a moment, this is to assume first-order metaphysical claims that we should not in this metametaphysical debate.

7 The same goes for the notion of 'fundamental'. If we think that the best language contains only those terms that refer to fundamental entities, then we cannot build that commitment into our the notion of semantic purpose.
} 
of economics, or the language of biology, or any other terms or language that we might adopt. If someone thinks that unrestricted mereological composition is correct, then a language that expresses those claims will be a language that they think satisfies the semantic purpose of representing objective similarities and differences in the world, and they will think that it does it better than a language that expresses the claims of mereological nihilism. To tell which is the best there has to be a shared semantic purpose. We, I argue, have that shared semantic purpose in virtue of the broad semantic purpose I have identified.

Is comparison relative to this broad semantic purpose possible? I think it is. To compare the language of physics to the language of economics is to ask which represents better the genuinely occurring similarities and differences in the world. If the language of physics represents better the objective similarities and differences then it is a better language relative to the metaphysically relevant broad semantic purpose; if the language of economics does, then it is better. To know this, though, we have to know, or at least put forward arguments about how the world really is, and what similarities and differences it contains.

That is, to make any claim about their relative goodness, including that they are equally good, requires first-order metaphysics of the sort that Irmak wants to resist. That is, Irmak must either accept that physics is a better candidate for being the privileged language, a worse candidate, or equally good. Each of these claims though rely on first-order metaphysical claims about what kinds of objective similarities and differences exist in the world. We must accept that there are substantive first-order metaphysical disputes in order to both accept and deny that there are equally good languages relative to the semantic purpose of representing all objective similarities and differences in the world. This form of deflationism can only support their claim that there are multiple equally good languages by making first-order metaphysical claims about the nature of the world.

Why, though, should we adopt this broader semantic purpose? Instead we might just think that it is enough that we introduced the language of physics on the grounds that it is an additional linguistic tool to understand, represent, and predict physical features of the world. After all, a language is a kind of social artefact that is created, maintained, and revised to fulfil various goals. The answer, I think, is in this last claim already. We have various goals with languages. Some are big, some small, and some of them will overlap with each other. I am not making the claim that we should all adopt this broader semantic purpose, or consider it when we speak. I am only claiming that it is this semantic purpose that the metaphysical realist intends to adopt. Others might find this purpose a boring one to adopt, but just because adopting a metaphysically relevant purpose will not be useful in other cases, or be seen as a fulfilling endeavour by all, does not result in the conclusion that such a semantic purpose does not exist. I am also not claiming that we, with our finite abilities, can create a language that fulfils this purpose. There may be reasons for thinking that we cannot. There might be epistemic reasons for thinking that 
we could never know if a language we have fulfils the broad semantic purpose I have identified. My claim has only been that what a metaphysician is doing when they introduce a putatively best language is to introduce a language that has the semantic purpose of representing all similarities and differences in the world. What determines the metaphysical goodness of a language is relative to this semantic purpose, not some narrower one. Likely there will be some cases still - some impasses - where we cannot decide which putative metaphysically best language is actually better. Such impasses will not always arise though, and the metametaphysical claim that the realist needs here is only that two languages have a shared semantic purpose against which they can be judged, contra Irmak's brand of deflationism.

\section{III}

The immediate response will be that I have somewhat missed the point. Two sets of propositions can be taken to share this broad purpose, but comparing them would still be impossible. After all, is a language that has expressed some aesthetic objective similarities better or worse than a language that has expressed some physical objective similarity? This ultimately the main deflationist claim that I am concerned with here.

However, this still relies on an assumption about the underlying metaphysical nature of reality. Take Sider again as an example. Sider discusses the view that for every aesthetic judgement there is a physical property $P$ that is the linguistic meaning of 'is beautiful' (Sider 2011: 58-59). This is a statement of his physicalism. Under this view, the physical language is metaphysically better because the aesthetic judgement can be reduced to the physical - the physical property is what is picked out by the predicate 'is beautiful'.

Physicalism, though, is a metaphysical commitment to the idea that, for example, aesthetic judgements can be reduced to statements about physical properties. But physicalism in this sense is a claim about what the objective language looks like, not about whether there is one. In order to conclude that two sets of propositions - those of physics and those of aesthetics for example - cannot be compared, we have to assume that one or both of these language is not the language that represents all objective similarities and differences in the world. We have to assume that there are no fundamentally aesthetic objective similarities and differences.

If we have a prior commitment to physicalism, the narrow semantic purpose representing the objective physical similarities and differences in the world and the broad semantic purpose of representing all objective similarities and differences in the world, collapse into each other. The purposes collapse into each other as we have antecedently assumed that all of the world's objective similarities and differences in the world are physical. But we of course need not accept this if our aim, as mine is, is to discuss the metaontological issues as opposed to the first-order metaphysical claims. 
The mistake lies in confusing the claim that physics is the objectively best language, and hence why it seems as though other languages must have a different semantic purpose, with an argument that defends that there is an objectively best language. Irmak says that the purpose of physics is to explain the fundamental physical structure of reality. But that physics has the purpose of explaining the fundamental physical structure of reality tells us nothing about the fundamental nature of reality unless we independently assume that the fundamental nature of reality is physical. This is clearly a first order claim, and other languages will be worse relative to the narrow purpose of explaining the physical structure of reality. But this is not the semantic purpose of a fundamental language, as the semantic purpose of a fundamental language cannot, prior to investigation, be assumed to only be interested in physical reality.

Answering whether a language that has expressed some aesthetic objective similarities is better or worse than a language that has expressed some physical objective similarity depends then on some underlying metaphysical claims about the nature of reality. Perhaps the language of aesthetics in fact best carves up the world. Whether this is the case is a matter of first-order debate within metaphysics about what joints in nature there are, what similarities and differences really exist, and what language best describes those that do exist. In our scientifically inclined world, many typically assume that it is physics that does this. We could, though, instead make arguments for aestheticalism, the view that the objective similarities and differences in the world are all fundamentally aesthetic in nature. If we thought this, then we presumably defend the idea that physical entities should be reduced to aesthetic ones, and thus that the aesthetic language is better than the physical language. Comparison of candidate privileged languages becomes an issue about what first order metaphysical claims, such as physicalism or aestheticalism, we wish to endorse.

A pluralistic metaphysics where there are many different types of joints in reality is not a denial of the claim that there is a fundamental language, or a language that is objectively best. It is a denial that physics (or any single language or theoretical scheme) ${ }^{8}$ alone can provide that objectively best language to represent all objective similarities and differences. That is, it is a denial of the first order commitment to physicalism. Many would be sympathetic to this claim, but it does not touch upon the truth (3) and (4). It may help illuminate that some physicalist might be making an assumption about what the content of the best language will be like, but not about there being such a fundamental language.

Perhaps, instead, we object that neither language is better. The concern might be that comparing language is trivial if the languages compared all successfully track similarities and differences, or do so equally well. Perhaps both the physical and aesthetic languages carve reality at objectively real joints. If this is the case, then it does not suggest that (3) is false. Rather, it suggests that the objectively best

\footnotetext{
8 In so far as physics is in some sense isolated from other sciences.
} 
language needs to be broad enough to include elements of both physical and aesthetic languages, contra Sider's first-order metaphysical claim that we should reduce aesthetic talk to physical properties, but in line with the metaontological realism Sider defends.

If all that we are interested in is arguments for and against the possibility of an objectively best language, then we should not antecedently adopt any metaphysical principle such as physicalism or aestheticalism that would dictate what that language's contents might be like. The argument of Sider's that Irmak focuses on is not entirely about whether (3) and (4) is correct, but also for (b): that the language of physics is the language which best carves nature at the joints. Once we separate these two aspects, and ignore for present purposes whether (b) is correct, we can see that (3) and (4) are not threatened by the notion of semantic purpose once we understand it, as the metaphysician does, to be coarse-grained.

There is, though, a broader point about different languages and the objectively best language, one that metaphysicians are perhaps too quick to ignore. It is that we should not, without good reason, rule out any language as potentially contributing to the objectively best language. Those good reasons are going to be arguments in favour or against of some metaphysical principle, such as physicalism. If we have good independent reasons to accept physicalism, then we can start to restrict which languages we think might contribute to the objectively best language. But for many it is far from clear that we have those reasons currently or that we could ever have those reasons. ${ }^{9}$ Progress is only going to be made on providing the content of the objectively best language once we recognise that a large part relies on the metaphysical principles that we take to be important to restricting its contents. If I have no good independently motivated reason for ruling out a language as being interested in representing objective similarities and differences in the world, then it must be considered as potential being (or contributing to) the objectively best language. That is what my toy example of aestheticalism was intended to show. Of course, providing arguments for or against metaphysical principles of the sort I have been discussing is difficult, and I can provide none here in favour of aestheticalism. ${ }^{10}$ But it does indicate the need for metaphysical debate, and why it is substantive, highly non-trivial debate (at least not for any reasons discussed here).

\section{IV}

My claim is that we can define the relevant semantic purpose of a language that purports to be objectively best or privileged is as being that of an attempt to represent all of the objective similarities and differences in the world. What, though, about a language that does not have this as a purpose at all?

\footnotetext{
9 Physicalism is, of course, a far from universally accepted claim. See, inter allia, Chalmers (1996), Chomsky (2000), Hempel (1969), Jackson (1986), and Kripke (1982) for just some of the arguments against the view.

10 Indeed, whilst the aesthetic language might be the best to use relative to the semantic purpose of talking about the beauty of artwork, it seems hard to believe that it would be sufficiently complete when comparing that language qua being a putative privileged language. This, though, is a first-order issue independent of my metaontological arguments in this paper.
} 
For example, take a language that by stipulation is introduced with a semantic purpose that does not include an attempt to describe objective similarities and differences in the world. ${ }^{11}$ It is unclear, though, why the realist about a metaphysically best language should be concerned with such languages. If they are not intended to be candidates for being the best language, then we simply have no need to consider them when we are seeing which language is the best candidate we have for being the metaphysically best language. Such languages, if there are any, might of course be highly useful and important for other reasons. They simply are not aiming to describe objective similarities and differences in the world. Nothing can be inferred from this as to their usefulness without some further claim about what pragmatically could be a useful language. The language that does not have this purpose should only be rejected if we are currently interested in representing those similarities and differences.

However, we must also remember here that economic, sociological, aesthetic languages, and ordinary language, are not like this language. At least part of their semantic purpose is to describe the objective similarities and differences in the world. Deflationists must hold this if they wish to maintain a belief in minimal realism, the view that the view that the world contains some similarities and differences that are entirely independent of us and our language choice, yet we nonetheless are trying to express those differences within our language and general inquiries into the world. This follows simply from the conjunction of (1) and (2) above. Most (if not all) language that we care about in our lives will therefore not be like the language that has no part of its semantic purpose as representing objective similarities and differences in the world. To compare the putative privileged languages, we must do so relative to the coarse-grained semantic purpose.

The conclusions of this paper, though, are more significant than this precisification of what semantic purpose is the relevant one. My ultimate point has been to argue that in order to know whether two putative privileged languages are equally good, relative to the coarse-grained semantic purpose I have identified, we have to engage in substantive first-order metaphysical disputes. In order to know whether the languages of physicalism and aestheticalism are equally good then we need to consider whether we think that there are objective physical and/or aesthetic similarities and differences in the world. To assess the languages to see which are the 'good' metaphysical languages requires us to assess the metaphysical principles that underlie those languages. It is this that ultimately undercuts the deflationist argument.

\footnotetext{
11 This cannot be just to say that that language is allegorical or metaphorical, as might be reasonable to suppose that even allegorical and metaphorical language are intended to describe some objective similarities and differences at least some of the time. If this was not the case, then those languages would not be useful in the way that they are at teaching children lessons about life, or highlighting some otherwise overlooked aspect of the world (assuming that they are useful in this way on at least some occasions). Some might hold that moral or modal talk is of this sort though. If I am a modal sceptic, then I could believe that no modal talk tracks any real distinction in the world, and that it is mere fiction.
} 
The deflationist faces a dilemma. Either languages do (at least in part) share the coarse-grained semantic purpose and so we can evaluate which language is better (even if the objectively best language might end up being a mixture of a number of somewhat different language), but to evaluate them requires first-order metaphysical commitments; or the languages do not have as any part of their semantic purpose the purpose of representing objective similarities and differences in the world in which case we have no reason to suppose that it was ever intended to be a metaphysically privileged language. I have provided no solution to what the metaphysically best language looks like, but hope to have shed some light on how we can decide which are languages are good.

\section{References}

Carnap, R., 1950/1956, 'Empiricism, Semantics and Ontology', Revue International de Philosophie 4: 20-40. Reprinted in Meaning and Necessity: A Study in Semantics and Modal Logic, 2nd edn. Chicago: University of Chicago Press.

Chalmers, D., 1996, The Conscious Mind, New York: Oxford University Press

Chalmers, D., 2009, ‘Ontological Indeterminacy'. In D. J. Chalmers, D. Manley, and R. Wasserman (eds.), Metametaphysics. Oxford: Oxford University Press. pp. 77-129.

Chomsky, N., 2000, New Horizons in the Study of Language and Mind, Cambridge: Cambridge University Press.

Hempel, C., 1969, 'Reduction: Ontological and Linguistic Facets', in S. Morgenbesser, et al. (eds.), Essays in Honor of Ernest Nagel, New York: St Martin's Press.

Hirsch, E., 2002a, ‘Against Revisionary Ontology', Philosophical Topics, 30(1): 103-127.

Hirsch, E., 2002b, ‘Quantifier Variance and Realism', Noûs, 36 (1): 51-73.

Hirsch, E., 2005, 'Physical-Object Ontology, Verbal Disputes, and Common Sense', Philosophy and Phenomenological Research 70: 67-97. Reprinted in Hirsch 2011: 144-77.

Hirsch, E., 2011, Quantifier Variance and Realism: Essays in Metaontology, New York: Oxford University Press.

Irmak, N., 2013, 'The privilege of the physical and the status of ontological debates', Philosophical Studies, Vol. 166(1): 1-18.

Jackson, F., 1986, 'What Mary Didn't Know', Journal of Philosophy, 83: 291-5.

Kripke, S., 1982, Wittgenstein on Rules and Private Language: An Elementary Exposition, Oxford: Basil Blackwell. 
Miller, J. T. M., 2016, 'Can an Ontological Pluralist Really be a Realist?', Metaphilosophy,47 (3): 425430.

Peacocke, C., 1988, 'The Limits of Intelligibility: A Post-Verificationist Proposal', Philosophical Review 97: 463-96.

Putnam, H., 1975, 'The Refutation of Conventionalism', in Putnam, Mind, Language and Reality: Philosophical Papers, volume 2, 153-91. Cambridge: Cambridge University Press.

Putnam, H., 1987, The Many Faces of Realism, La Salle, IL: Open Court.

Sidelle, A., 2002, 'Is there a True Metaphysics of Material Objects?', Philosophical Issues, 12: Realism and Relativism.

Sider, T., 2011, Writing the Book of the World, New York: Oxford University Press.

Sosa, E., 1999, 'Existential Relativity', in P. French and H. K. Wettstein (eds.), Midwest Studies in Philosophy XXIII: New Directions in Philosophy, 132-43. Oxford: Blackwell.

Thomasson, A. L., 2007, Ordinary Objects. New York: Oxford University Press.

Thomasson, A. L., 2008, ‘Existence Questions’, Philosophical Studies, 141: 63-78.

Thomasson, A. L., 2009, 'Answerable and Unanswerable Questions', in Chalmers et al., 2009, 444-71.

Thomasson, A. L., 2015, Ontology Made Easy, New York: Oxford University Press. 